\title{
Aerodynamic Analysis of the Truss-Braced Wing Aircraft Using Vortex-Lattice Superposition Approach
}

\author{
Eric Ting * \\ Stinger Ghaffarian Technologies Inc., Moffett Field, CA 94035 \\ Kevin Reynolds ${ }^{\dagger}$ \\ NASA Ames Research Center, Moffett Field, CA 94035 \\ Nhan Nguyen $*$ \\ NASA Ames Research Center, Moffett Field, CA 94035 \\ Joseph Totah ${ }^{\S}$ \\ NASA Ames Research Center, Moffett Field, CA 94035
}

\begin{abstract}
The SUGAR Truss-Braced Wing (TBW) aircraft concept is a Boeing-developed $\mathrm{N}+3$ aircraft configuration funded by NASA ARMD Fixed Wing Project. This future generation transport aircraft concept is designed to be aerodynamically efficient by employing a high aspect ratio wing design. The aspect ratio of the TBW is on the order of 14 which is significantly greater than those of current generation transport aircraft. This paper presents a recent aerodynamic analysis of the TBW aircraft using a conceptual vortex-lattice aerodynamic tool VORLAX and an aerodynamic superposition approach. Based on the underlying linear potential flow theory, the principle of aerodynamic superposition is leveraged to deal with the complex aerodynamic configuration of the TBW. By decomposing the full configuration of the TBW into individual aerodynamic lifting components, the total aerodynamic characteristics of the full configuration can be estimated from the contributions of the individual components. The aerodynamic superposition approach shows excellent agreement with CFD results computed by FUN3D, USM3D, and STAR-CCM+.
\end{abstract}

\section{Introduction}

Demand for green aviation is expected to increase with the need for reduced environmental impact. Most large transports today operate within the best cruise L/D range of 18-20 using the conventional tube-and-wing design. This configuration has led to marginal improvements in aerodynamic efficiency over this past century, as aerodynamic improvements tend to be incremental. A big opportunity has been shown in recent years to significantly reduce structural weight or trim drag, hence improved energy efficiency, with the use of lightweight materials such as composites. The Boeing 787 transport is an example of a modern airframe design that employs lightweight structures. High aspect ratio wing design can provide another opportunity for further improvements in energy efficiency.

Historically, the study of high aspect ratio wings has been intimately tied to the study of aeroelasticity and flutter. These studies have sought to develop tools and methods to analyze aeroelastic effects by laying the foundation for more modern high aspect ratio wing aircraft such as the Truss-Braced Wing (TBW).${ }^{1-3}$ Originally suggested by Northrop Grumman for the development of a long-range bomber, the idea of using truss structures to alleviate the bending moments of an ultra-high aspect ratio wing has culminated in more than a decade of work focused on understanding the aeroelastic properties and structural weight penalties due to the more aerodynamically efficient wing.

The Subsonic Ultra Green Aircraft Research (SUGAR) Truss-Braced Wing (TBW) aircraft concept is a Boeingdeveloped N+3 aircraft configuration funded by NASA ARMD Fixed Wing Project. ${ }^{4,5}$ The TBW aircraft concept is designed to be aerodynamically efficient by employing an aspect ratio on the order of 14 , which is significantly greater than those of conventional aircraft wings. As a result, intermediate structural supports are required. The main wings

\footnotetext{
*Stinger Ghaffarian Technologies Inc., NASA Ames Research Center, Research Engineer, eric.b.ting@ nasa.gov

$\dagger$ NASA Ames Research Center, Aerospace Engineer, kevin.w.reynolds@nasa.gov

¥NASA Ames Research Center, Research Scientist, AIAA Associate Fellow, nhan.t.nguyen@nasa.gov

${ }^{\S}$ NASA Ames Research Center, Research Scientist, AIAA Associate Fellow, joseph.j.totah@nasa.gov
} 
are braced at approximated mid-span by two main trusses. In addition, two jury struts, one on each wing, provide additional reinforcement. Figure 1 is an illustration of the TBW aircraft.

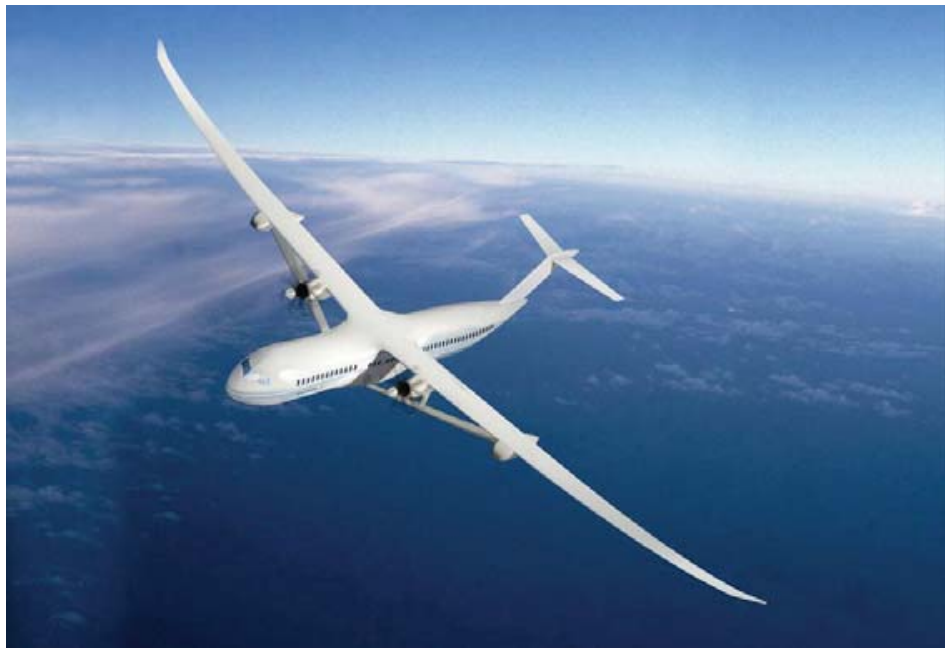

Figure 1. Boeing SUGAR Truss-Braced Wing (TBW) Aircraft Concept

The development of the TBW aircraft is supported through a collaboration between the NASA Fixed Wing Project, Boeing Research and Technology, and a number of other organizations. Multidisciplinary design analysis and optimization (MDAO) studies have been conducted at each stage to improve the wing aerodynamics, structural efficiency, and flight performance using advanced $\mathrm{N}+4$ turbofan engines. These MDAO studies have refined the geometry of the wing and configuration layout and have involved trade studies involving minimizing induced drag with wing span, minimizing profile drag at lower Reynolds numbers, and minimizing wave drag due to the addition of the strut and brace. The chart in Fig. 2 summarizes progression of the past revisions of the TBW aircraft design at various developmental stages. ${ }^{4,5}$
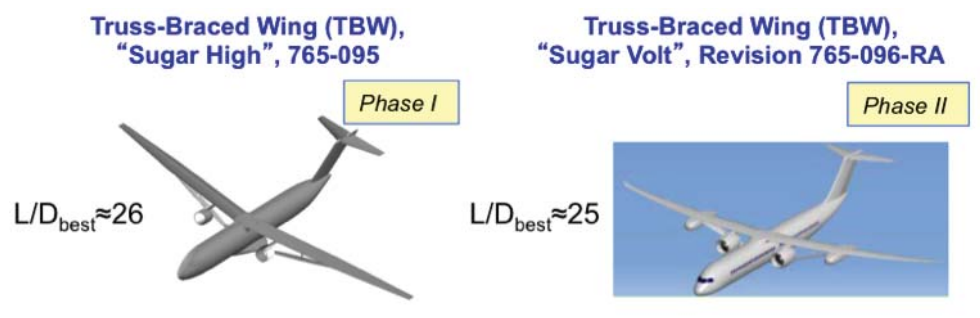

\begin{tabular}{|l|l|}
\hline Mach & 0.7 \\
\hline Max Range [nm] & 3,500 \\
\hline Wing Area [sft] & 1,722 \\
\hline Aspect Ratio & 26.94 \\
\hline Wing Span [ft] & 215 \\
\hline MAC [ft] & 9.19 \\
\hline Cruise CL & 0.77 \\
\hline Init. Cruise Altitude [ft] & 42,000 \\
\hline Sweep @ c/4 ['] & 8.02 \\
\hline TGOW [lb] & 176,800 \\
\hline SLS Thrust/engine [lb] & 32,641 \\
\hline
\end{tabular}

\begin{tabular}{|l|l|}
\hline Mach & 0.7 \\
\hline Max Range [nm] & 3,500 \\
\hline Wing Area [sft] & 1,477 \\
\hline Aspect Ratio & 19.55 \\
\hline Wing Span [ft] & 169.94 \\
\hline MAC [ft] & 9.19 \\
\hline Cruise CL & 0.81 \\
\hline Init. Cruise Altitude [ft] & 42,000 \\
\hline Sweep @ c/4 [ ${ }^{\circ}$ ] & 12.52 \\
\hline TGOW [lb] & 150,000 \\
\hline SLS Thrust/engine [lb] & 35,000 \\
\hline
\end{tabular}

Figure 2. Summary of TBW Aircraft Development under NASA Phase I and II Contracts 
In the present, current research into the TBW as a future generation aircraft is being conducted. Owing to its high aspect ratio and highly flexible, modern material technology based wings, significant aeroelastic interactions are expected for the aircraft. Aeroelastic interactions can result in adverse aerodynamics on the aircraft and lead to drag increases. The TBW also represents a N+3 testbed for the evaluation of adaptive aeroelastic shape control (AASC) technologies such as the Variable Camber Continuous Trailing Edge Flap (VCCTEF) system ${ }^{6,7}$ currently investigated under the AASC element of the Fixed Wing project by NASA, Boeing, and partners. A MDAO framework is currently being developed for analyzing future generation aircraft configurations such as the TBW and the application of AASC technologies including the VCCTEF. This framework is intended to include a suite of aerodynamic tools of varying fidelity, as well as finite-element models of these configurations.

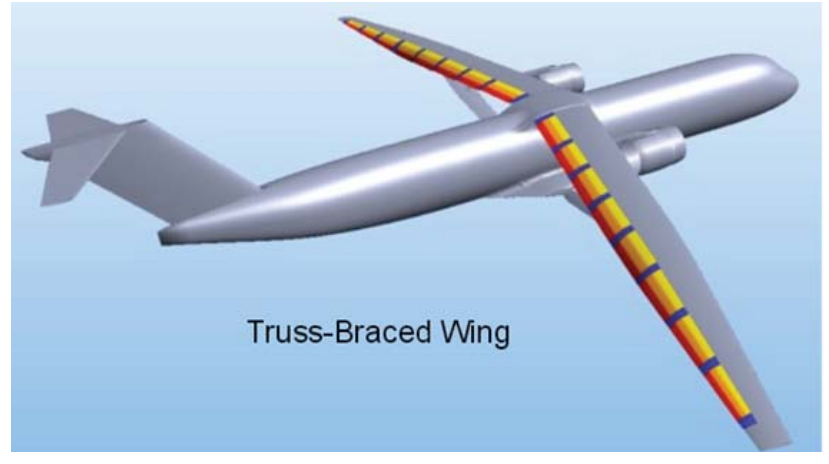

Figure 3. Notional Diagram of TBW with VCCTEF System

This paper presents an initial aerodynamic analysis of the TBW aircraft using a conceptual vortex-lattice aerodynamic tool VORLAX coupled with the aerodynamic superposition method. Based on the underlying linear potential flow theory, the complex configuration of the TBW is modeled using superposition of individual aerodynamic components to estimate the total aircraft characteristics. This is an approximate method for conceptual preliminary analysis, which does not fully address aerodynamic interference effects occurring at the interfaces of these components. This conceptual aerodynamic method is being developed as an initial analysis capability for the MDAO framework of the TBW.

\section{Initial Preliminary Aerodynamic Analysis}

Preliminary aerodynamic analysis was conducted in a vortex-lattice environment using the vortex-lattice code VORLAX, an aerodynamic performance prediction code developed by Miranda et al. ${ }^{8}$ Based on aerodynamic lifting line and vortex-lattice method (VLM), VORLAX provides a rapid method for estimating force and moment coefficients as well as stability and control derivatives of an aerodynamic configuration. An aerodynamic configuration is constructed within VORLAX by a series of lifting panels that are formed by spanwise and chordwise locations of horseshoe vortices based on a lattice discretization specified by the user. VORLAX then computes the vehicle aerodynamics in both the longitudinal and lateral directions independently. The longitudinal and lateral computational results are combined to produce overall aerodynamic characteristics of an aerodynamic configuration at any arbitrary angles of attack and sideslip. Graphical user interfaces (GUIs) have been developed for VORLAX such as VORVIEW and Vehicle Sketch Pad (VSP), ${ }^{9}$ which are front end programs that allow users to create a model and execute VORLAX interactively or in a batch mode.

Because VLM is based on potential flow theory, it is an inviscid code that can only compute inviscid drag due to lift and cannot predict viscous or wave drag. The compressibility effect on the aerodynamic coefficients is accounted for by a compressibility correction, such as the Prandtl-Glauert correction. For lift prediction at low angle of attacks, VORLAX can provide reasonably accurate prediction. Figure 4 shows a comparison between the lift coefficient predicted by VORLAX and the measured lift coefficient of a sub-scale 5.5\% wind tunnel model of the NASA Generic Transport Model (GTM) ${ }^{10}$ tested in NASA Langley Research Center's 14-Foot-By-22-Foot Wind Tunnel. The agreement between the VORLAX lift prediction and test data is excellent. 


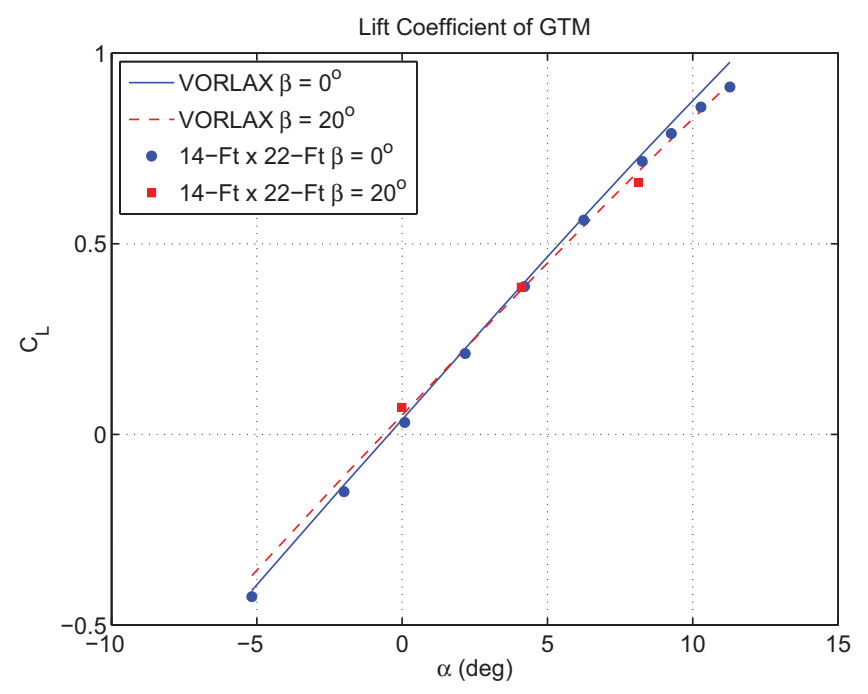

Figure 4. GTM Lift Coefficient Comparison between VORLAX and Wind Tunnel Test Data

Aerodynamic models of the TBW with varying degree of fidelity already exist, including a FUN3D model by Robert Bartels at NASA Langley Research Center and a USM3D model by Richard Campbell at NASA Langley Research Center. During an initial analysis, a full configuration of the TBW was created in VORLAX, and the mesh and an example panel/vortex-lattice discretization for the TBW is shown in Fig. 5. The aerodynamic characteristics of the TBW were computed in VORLAX and then compared against FUN3D and USM3D results as shown in Fig. 6. It can be seen that the VORLAX results do not agree well with CFD results.
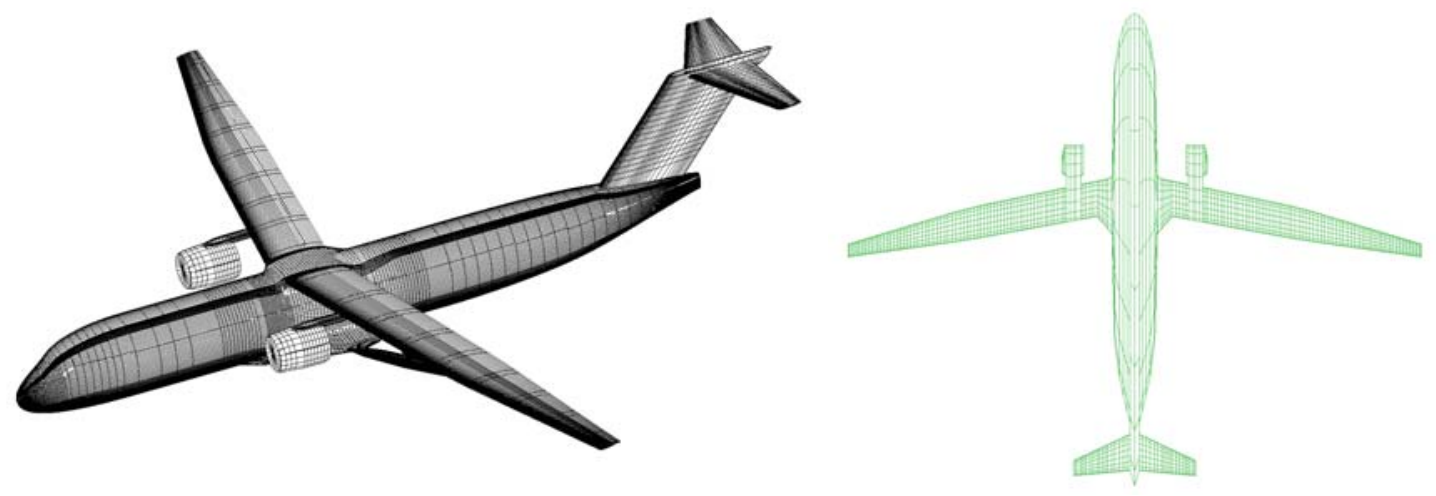

Figure 5. TBW Mesh (Left) and Vortex-Lattice Panel/Polygon Discretization (Right) in VORVIEW

Subsequently, a high-fidelity commercial CFD code STAR-CCM+ was used by John Melton at NASA Ames Research Center to analyze the TBW configuration. The STAR-CCM+ model has 23 million volume cells and 572,000 surface triangles over the left half of the TBW, as shown in Fig. 7. The solver computed steady-state solutions using the SST $(k-\omega)$ turbulence model. Figure 8 shows the lift curve predicted by STAR-CCM+, which has a lift curve slope $C_{L_{\alpha}}=10.142$ at Mach 0.7 and $2^{\circ}$ angle of attack. The results agree well with the FUN3D and USM3D results. 

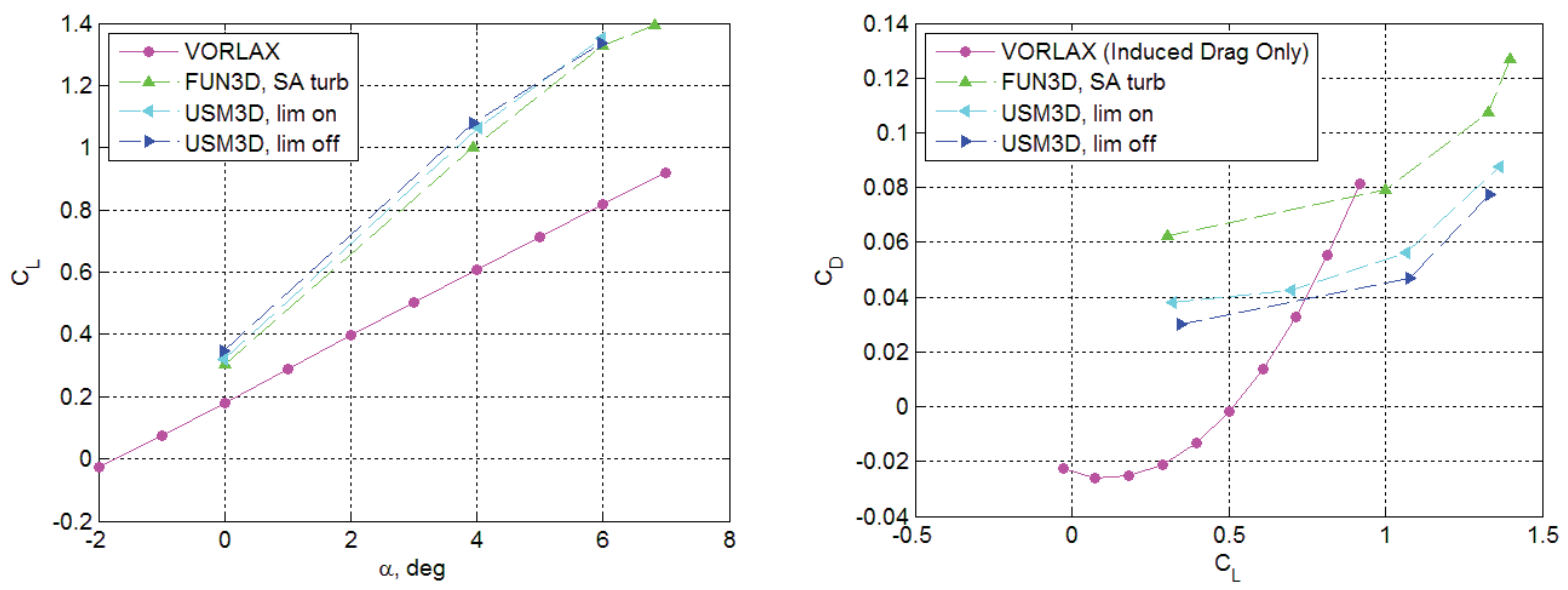

Figure 6. Comparison of TBW Lift Curve and Drag Polar from VORLAX, USM3D, and FUN3D

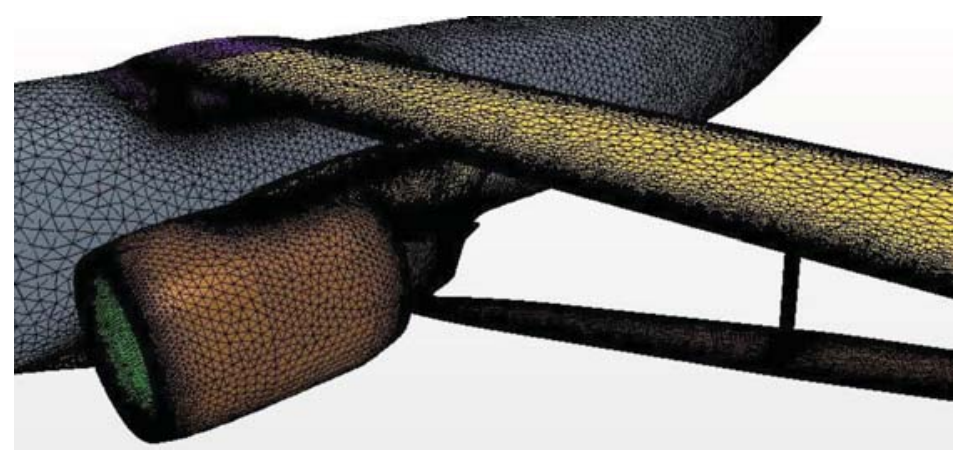

Figure 7. STAR-CCM+ Surface Triangulation of TBW

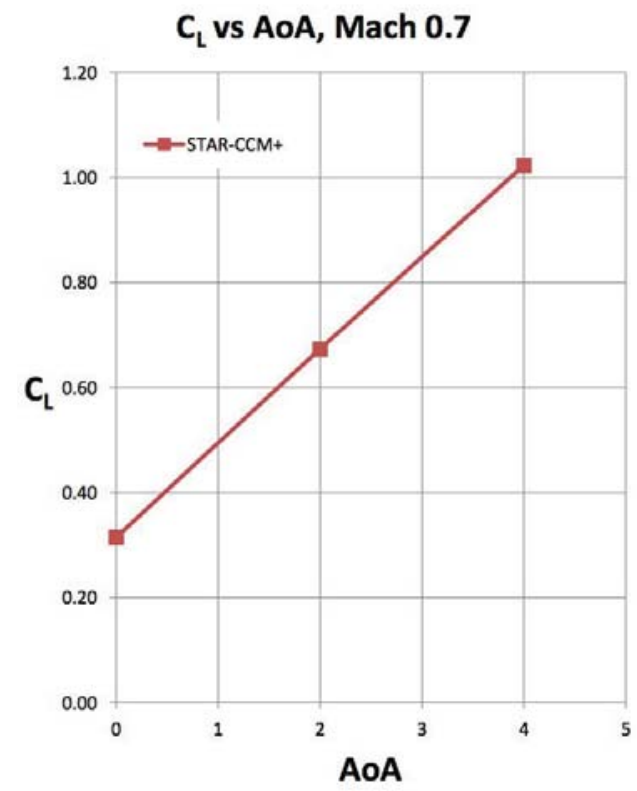

Figure 8. TBW Lift Curve Computed by STAR-CCM+ 
More interestingly, the lift curve slope obtained by integrating the surface pressure distribution over the wing alone is $C_{L_{\alpha}}=7.688$, which is $20 \%$ less than the lift curve slope for the full configuration. The significant difference between the full configuration and the wing-alone lift curve slopes suggested that the trusses may be generating a significant amount of lift that was not correctly captured by VORLAX due to the nature of the mean camber vortex sheet analysis. In fact, it is observed from Fig. 9 that the trusses, or braces, show a strong suction region near the leading edge. This observation led to an alternate approach of vortex-lattice modeling based on the principle of aerodynamic superposition.

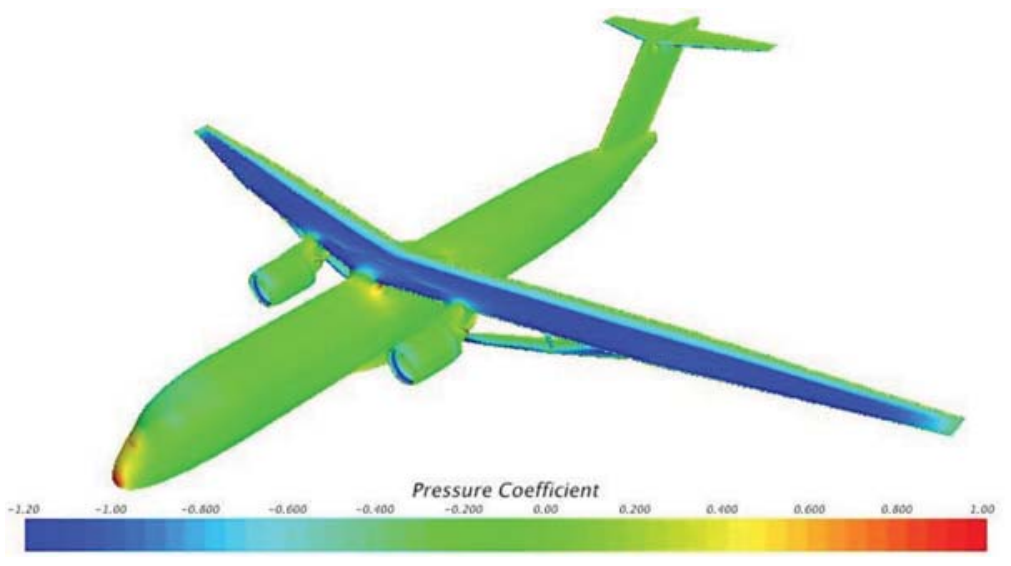

Figure 9. TBW Pressure Coefficient Distribution Computed by STAR-CCM+

\section{Aerodynamic Superposition Vortex-Lattice Approach}

Vortex-lattice method models a lifting aerodynamic surface as a vortex sheet formed by the mean camber surface. It generally provides reliable aerodynamic prediction for a simple lifting aerodynamic surface such as a cantilever wing. However, for complex configurations such as the TBW where multiple lifting surfaces are present and located in close proximity in the streamwise direction, the standard modeling approach in VLM can break down. This has been demonstrated by the previous results of the full configuration VORLAX model of the TBW in Fig. 6.

Because of its basis in potential flow theory for inviscid, incompressible, irrotational flow, VLM uses velocity potential functions to effectively compute harmonic solutions of the Laplace's equation. Since the Laplace's equation is a linear second-order partial differential equation, the principle of superposition of aerodynamic solutions holds. This aerodynamic superposition principle can be used as an alternative approach to analyzing complex aerodynamic configurations, and is applied to the analysis of the TBW.

Using the aerodynamic superposition principle, the lifting surfaces of the TBW are analyzed individually and then their contributions to the aerodynamic forces and moments are summed together to build up the aerodynamic characteristics of the full configuration of the TBW. This approach splits up the full configuration of the TBW into components. Because they are not considered a lifting surfaces, the engines and pylons are removed from the vortex-lattice models as a simplification. ${ }^{11}$ The jury struts are also omitted from consideration. Two aerodynamic superposition methods are considered:

- Method 1 - The TBW full configuration is broken up into three components: 1) fuselage-wing-tail empennage component, 2) fuselage-brace-tail empennage component, and 3) fuselage-tail empennage component. The full configuration is the sum of the fuselage-wing-tail component and fuselage-brace-tail empennage component minus the fuselage-tail empennage component. This is illustrated in Fig. 10. This approach was proposed as a way to capture the lifting contribution from the braces without completely eliminating any of the lifting line interference effects that is captured by VORLAX. 

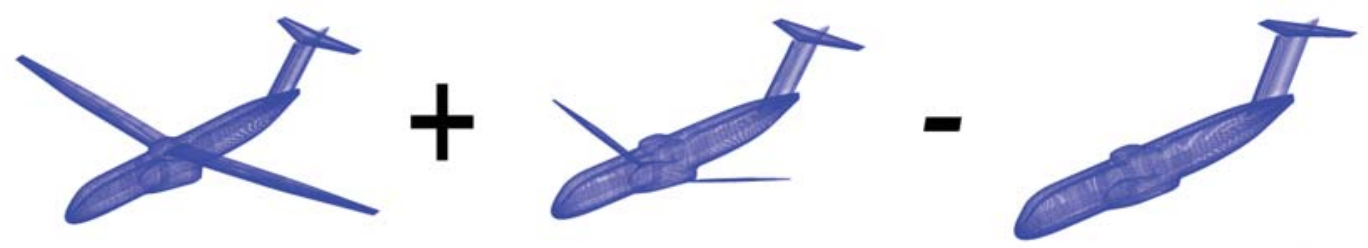

Figure 10. Aerodynamic Superposition Method 1 for TBW Model

- Method 2 - The TBW full configuration is broken up into four components: 1) fuselage component, 2) wing component, 3) brace component, and 4) horizontal tail component. The full configuration is the sum of all individual components. The vertical tail is not included since it does not contribute to the aircraft lift in symmetric flight. This is illustrated in Fig. 11. This approach reduces the TBW configuration into solely lifting surfaces, and each component is examined without interference from the others to minimize any adverse modeling simplifications that VLM may be imposing.

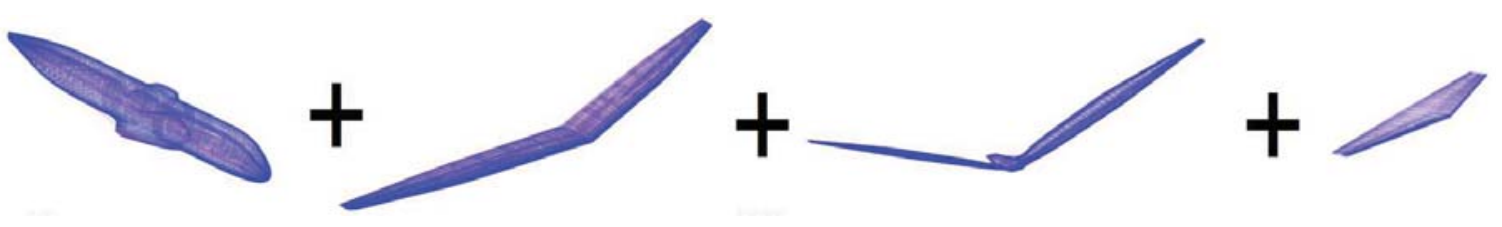

Figure 11. Aerodynamic Superposition Method 2 for TBW Model

Since VLM can only compute induced drag, viscous skin friction drag is estimated and then added to the inviscid drag to provide a rough estimate of the total drag without the wave drag contribution, which otherwise could be computed using the Korn's equation.

The following relationships form the basis for approximating viscous skin friction drag as described by Abbott and Von Doenhoff: ${ }^{12}$

$$
\begin{aligned}
C_{D_{f, \text { wing }}} & =k c_{f} \frac{S_{w_{\text {wing }}}}{S_{\text {ref }}} \\
C_{D_{f, \text { fuselage }}} & =K c_{f} \frac{S_{w_{\text {fuselage }}}}{S_{\text {ref }}}
\end{aligned}
$$

The key parameters in these expressions are the calculation of skin friction coefficient, $c_{f}$, and the corresponding form factors for airfoil, $k$, and fuselage, $K$, shapes. The airfoil form factor $k$ is given by reference ${ }^{13}$ as a function of the thickness-to-chord ratio $t / c$, Mach number $M$, and wing sweep angle $\Lambda$. The fuselage (body) form factor $K$ is given by reference ${ }^{14}$ as a function of the fineness ratio $l / d$ and Mach number $M$.

The calculation of skin friction coefficient associated with the wing and fuselage is performed using flat plate approximation. The resulting expression for $c_{f}$ that captures the effect of laminar-to-turbulent flow transition is as follows:

$$
c_{f}=\frac{x_{c}}{\bar{c}}\left(1.328 R e_{x_{c}}^{-0.5}\right)+\left(0.072 R e_{\bar{c}}^{-0.2}\right)-\frac{x_{c}}{\bar{c}}\left(0.072 R e_{x_{c}}^{-0.2}\right)
$$

where $R e_{\bar{c}}$ is the Reynolds number based on the mean aerodynamic chord.

To estimate the transition length, $x_{c}$, which is the distance from the leading edge of the wing or nose of the fuselage where the flow transitions from laminar to turbulent flow, the following expression is used:

$$
x_{c}=\frac{R e_{x_{c}} \mu}{\rho V}
$$

where the value of $R e_{x_{c}}$ is the transition Reynolds number, $\mu$ is the kinematic (absolute) viscosity, $\rho$ is the density, and $V$ is the airspeed.

For varying values of $R e_{X_{c}}$ and airspeed at a given altitude, this expression yields the transition lengths depicted in Fig. 12. 


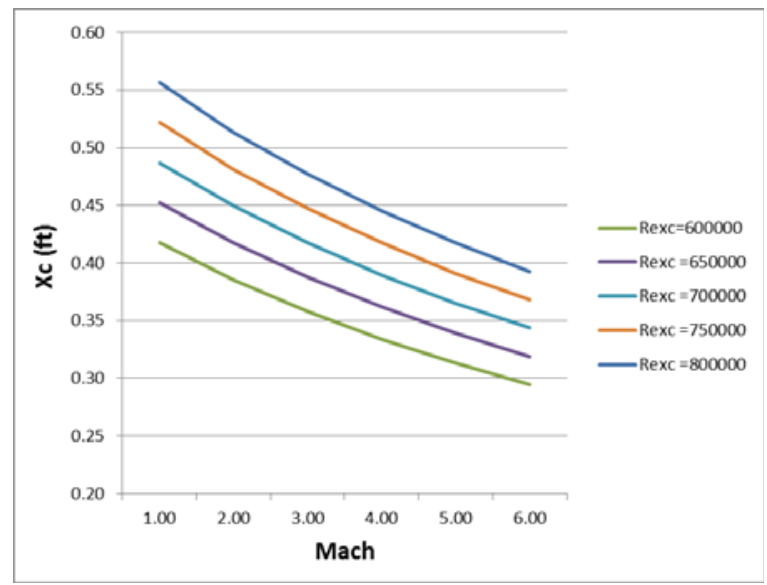

Figure 12. Transition Length Variation at $\mathbf{3 5 , 0 0 0} \mathrm{ft}$ Altitude (Standard Day)

The viscous skin friction drag correlation method is applied to the DLRF6 reference aircraft for validation. ${ }^{15}$ As can be seen in Fig. 13, VLM alone underestimates the drag for the DLRF6 reference aircraft. However, when viscous effects are added to the vortex-lattice drag estimates, the results appear much more reasonable, especially within an angle-of-attack range near the minimum drag point.

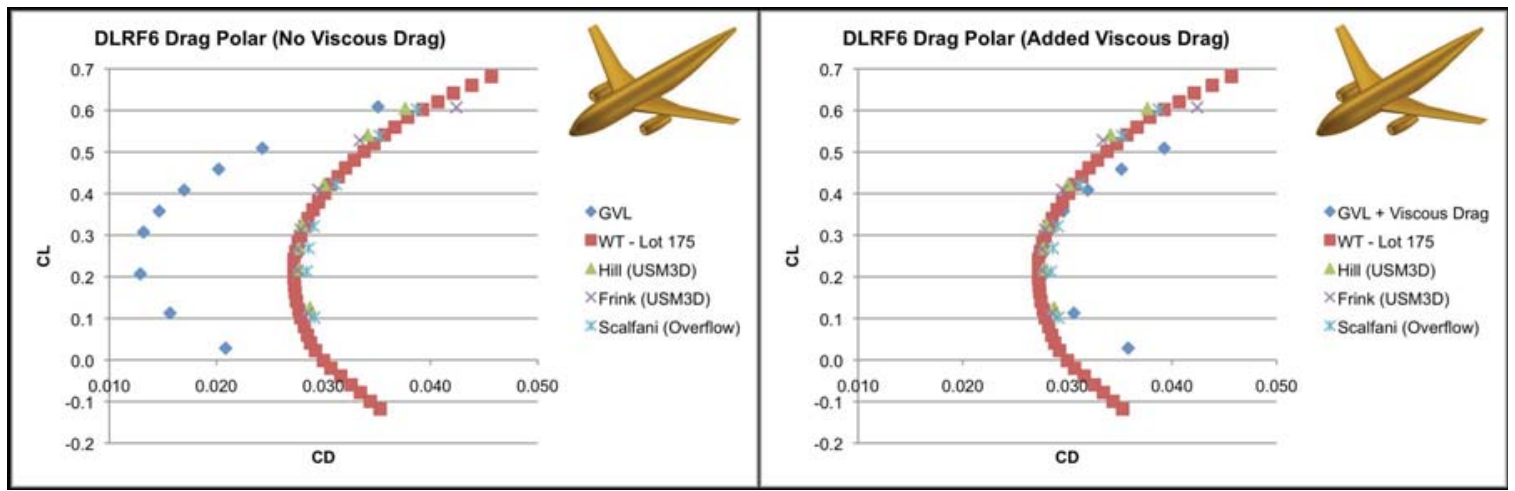

Figure 13. Preliminary Comparison of Drag Estimates with Previously Published Data for the DLRF6 Reference Aircraft

Using the viscous skin friction drag correlation method, the transition Reynolds number can be estimated if the transition location is known or computed from a high-fidelity CFD code. CFD analysis has shown that the TBW configuration can achieve significant natural laminar flow over the wings. The transition has been estimated by Richard Campbell at NASA Langley Research Center using USM3D RANS solver and the transition analysis tool LASTRAC. Laminar flow over the TBW wing is achieved up to $50 \%$ of the chord location at the design cruise condition of Mach 0.7 and 42,000 ft. The Reynolds number based on the mean aerodynamic chord (MAC) of the TBW is $R e=11.1 \times 10^{6}$ at the cruise condition. Thus, a critical transition Reynolds number of $R e_{x_{c}}=5.55 \times 10^{6}$ is used to calculate skin friction drag for all components. The critical length is approximately $x_{c}=4.57 \mathrm{ft}$ at a cruise condition of Mach 0.7 and altitude of $42,000 \mathrm{ft}$ corresponding to transition at $50 \%$ of the MAC. Table 1 summarizes the viscous skin friction drag estimates for the TBW at various flight conditions. 


\begin{tabular}{|c|c|c|c|c|c|c|c|}
\hline \multicolumn{9}{|c|}{$C_{D_{0}}$ (count) } \\
\hline Mach & 0.2 & 0.3 & 0.4 & 0.5 & 0.6 & 0.7 & 0.8 \\
\hline Altitude (ft) & 0 & 8,400 & 16,800 & 25,200 & 33,600 & 42,000 & 50,400 \\
\hline \hline Wing & 33 & 34 & 34 & 33 & 32 & 30 & 25 \\
\hline Brace & 3 & 4 & 4 & 3 & 3 & 3 & 3 \\
\hline Tail Empennage & 12 & 12 & 12 & 12 & 11 & 10 & 7 \\
\hline Fuselage & 53 & 52 & 52 & 53 & 54 & 55 & 57 \\
\hline Total & 101 & 102 & 102 & 101 & 100 & 98 & 92 \\
\hline
\end{tabular}

Table 1. Viscous Drag Estimates for TBW at Various Different Flight Conditions ( 1 count $=0.0001)$

\section{A. Aerodynamic Superposition Results}

The results for running VORLAX with the full configuration of the TBW and the results for running the TBW using the aerodynamic superposition methods are compared against the FUN3D and USM3D results, as shown in Fig. 12 and Table 2. The values of $C_{L_{0}}$ and $C_{L_{\alpha}}$ for the different aerodynamic modeling results are also summarized in Table 2, where a linear fit is conducted using the linear regions in the CFD results (approximately $\alpha \leq 5^{\circ}$ ).
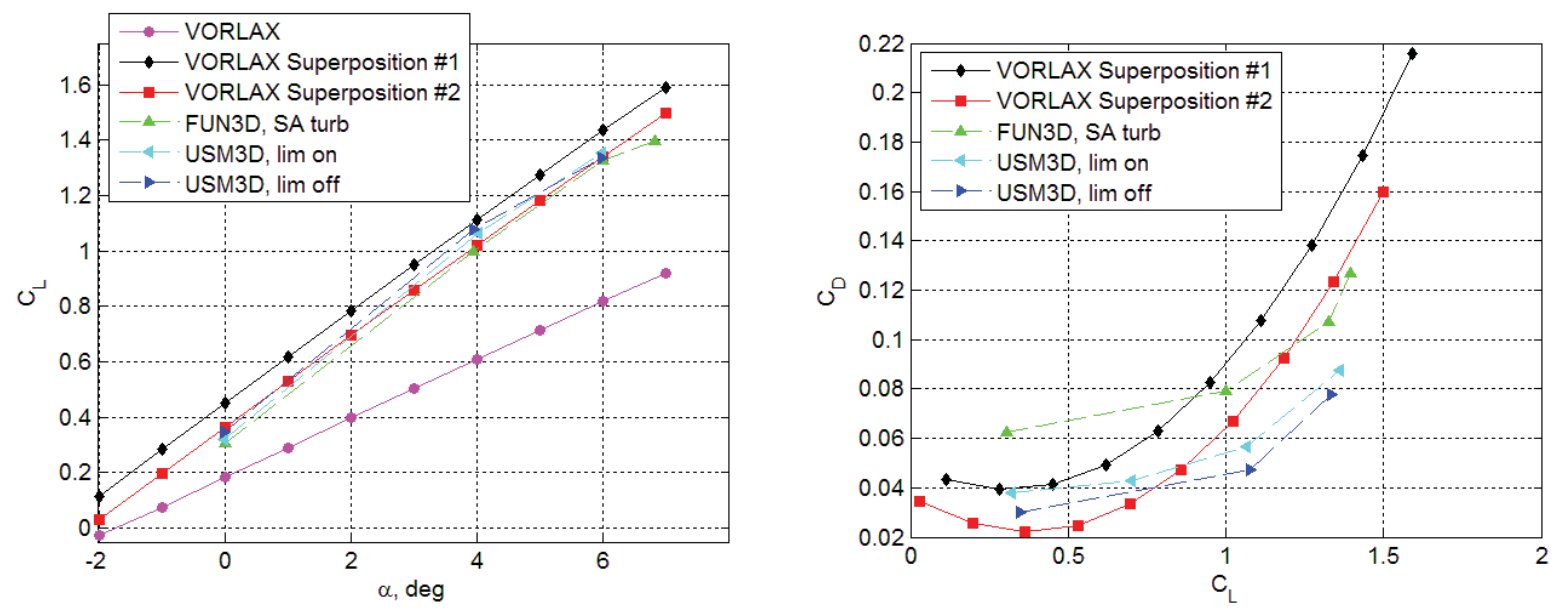

Figure 14. TBW Lift Curve and Drag Polar Comparison Between VORLAX Superposition Results Against CFD Results

\begin{tabular}{|c|c|c|c|c|}
\hline & $C_{L_{\alpha}}$ & $C_{L_{\alpha}} \%$ Difference & $C_{L_{0}}$ & $C_{L_{0}} \%$ Difference \\
\hline \hline FUN3D & 9.8225 & 1.59 & 0.3096 & 6.55 \\
\hline USM3D (lim off) & 9.9807 & 0 & 0.3313 & 0 \\
\hline USM3D (lim on) & 9.6230 & 3.58 & 0.3650 & -10.17 \\
\hline VORLAX (Full Configuration) & 6.0526 & 39.36 & 0.1828 & 44.82 \\
\hline VORLAX (Aerodynamic Superposition 1) & 9.4097 & 5.72 & 0.4504 & -35.95 \\
\hline VORLAX (Aerodynamic Superposition 2) & 9.3707 & 6.11 & 0.3629 & -9.54 \\
\hline
\end{tabular}

Table 2. TBW Lift Curve Comparisons

As can be observed, excellent agreement in the lift curve between the VORLAX aerodynamic superposition method and the CFD results is demonstrated. The largest difference in the lift curve slope between the VORLAX aerodynamic superposition and the CFD methods is about $6 \%$, while superposition method \#2 is able to more closely estimate $C_{L_{0}}$ in comparison to superposition method \#1. The drag polar predicted by the VORLAX aerodynamic superposition methods are compared against the USM3D results. For superposition method \#2, agreement between the 
two drag polars occurs at $C_{L}$ of about 0.8 . The drag polar for the full configuration VORLAX model is shown to have extremely poor agreement in Fig. 6 and thus is not shown in the figure.

\section{Component Aerodynamic Results for Superposition Method \#1}

Since the aerodynamic superposition approach provides aerodynamic contributions of the individual components, it is of interest to compare the contributions of these components to the overall aerodynamic characteristics of the TBW. The lift and drag values from VORLAX for the components of the first superposition method \#1 are shown in Figs. $15-18$.
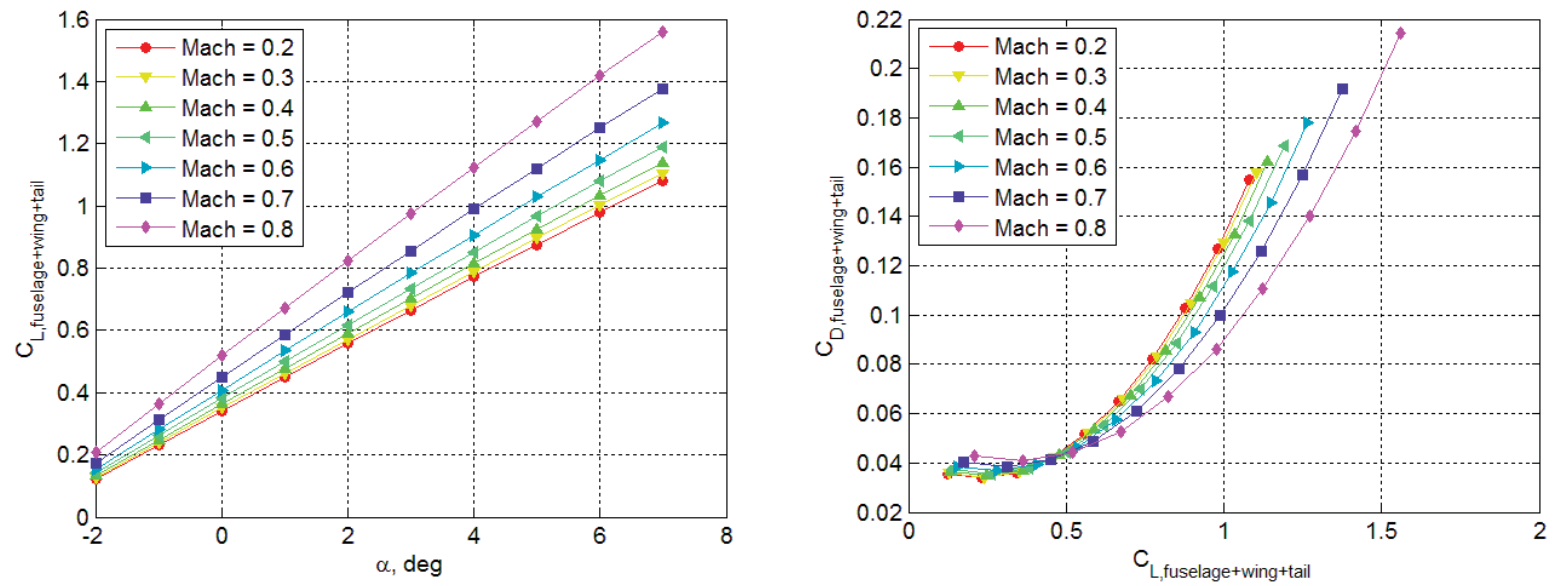

Figure 15. VORLAX Lift Curves and Drag Polars for TBW Fuselage+Wing+Tail
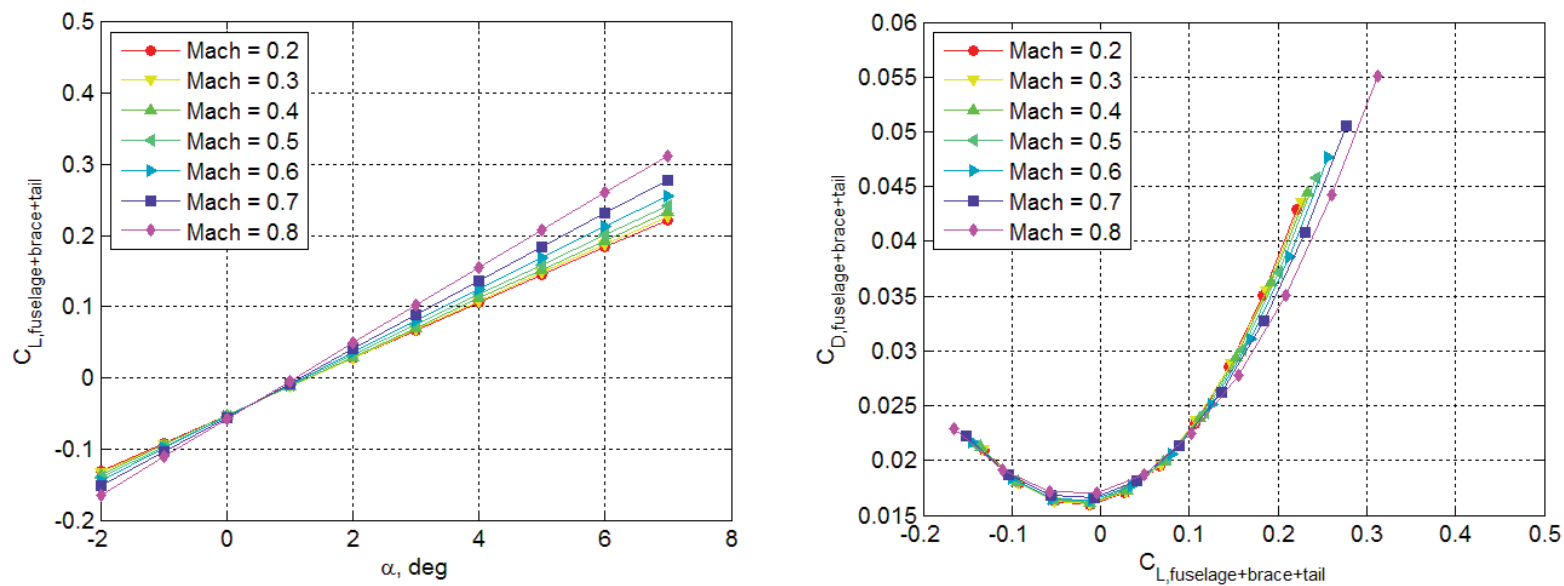

Figure 16. VORLAX Lift Curves and Drag Polars for TBW Fuselage+Brace+Tail 

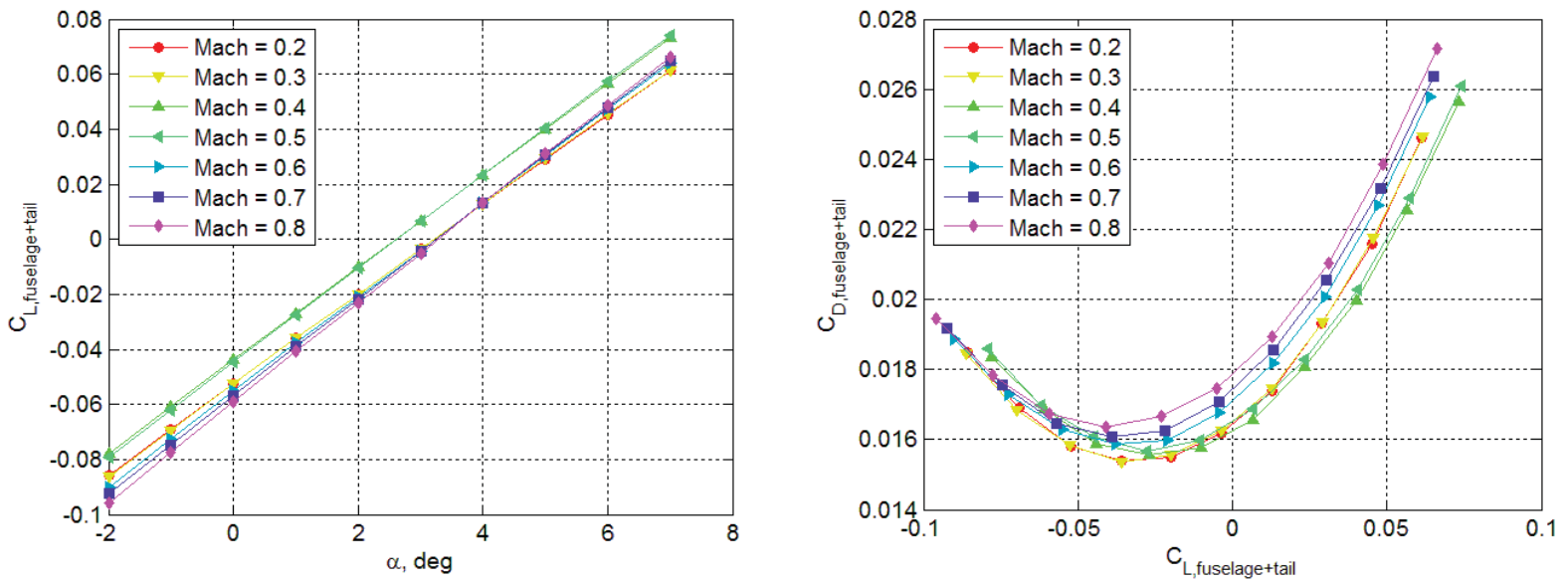

Figure 17. VORLAX Lift Curves and Drag Polars for TBW Fuselage+Tail
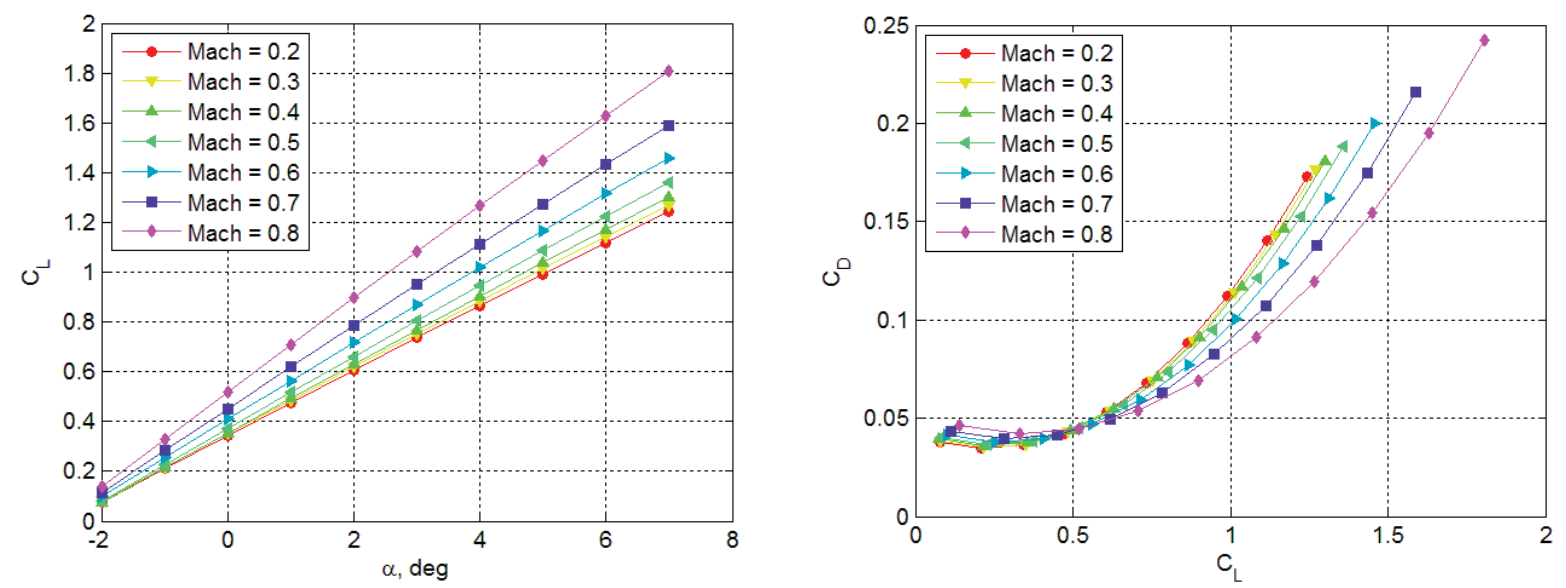

Figure 18. VORLAX Lift Curves and Drag Polars for TBW Full Configuration, Superposition Method \#1

Analysis of the VORLAX results using the superposition approach at the design cruise condition of $C_{L}=0.810$ produces a corresponding angle of attack of $\alpha=2.153^{\circ}$. The lift and drag contributions of the different components of the TBW using superposition method \#1 are computed and shown in Tables 3, 4, and 5.

\begin{tabular}{|c|c|c|}
\hline & Component $C_{L}$ & \% Contribution \\
\hline \hline Fuselage+Wing+Tail Empennage & 0.7428 & 91.70 \\
\hline Fuselage+Brace+Tail Empennage & 0.0480 & 5.93 \\
\hline -(Fuselage+Tail Empennage) & 0.0192 & 2.37 \\
\hline Total & 0.810 & 100 \\
\hline
\end{tabular}

Table 3. VORLAX Lift Contribution at Cruise $C_{L}=0.810$, Superposition Method \#1 $(1$ count $=0.0001)$ 


\begin{tabular}{|c|c|c|}
\hline & Component Induced $C_{D}$ (count) & \% Contribution \\
\hline \hline Fuselage+Wing+Tail Empennage & 543 & 96.45 \\
\hline Fuselage+Brace+Tail Empennage & 119 & 21.14 \\
\hline -(Fuselage+Tail Empennage) & -99 & -17.58 \\
\hline Total & 563 & 100 \\
\hline
\end{tabular}

Table 4. VORLAX Induced Drag Contribution at Cruise $C_{L}=0.810$, Superposition Method \#1 $(1$ count $=0.0001)$

\begin{tabular}{|c|c|c|}
\hline & Component Total $C_{D}$ (count) & \% Contribution \\
\hline \hline Fuselage+Wing+Tail Empennage & 638 & 96.52 \\
\hline Fuselage+Brace+Tail Empennage & 187 & 28.29 \\
\hline -(Fuselage+Tail Empennage) & -164 & -24.81 \\
\hline Total & 661 & 100 \\
\hline
\end{tabular}

Table 5. Total Estimated Drag Contribution at Cruise $C_{L}=0.810$, Superposition Method \#1 $(\mathbf{1}$ count $=\mathbf{0 . 0 0 0 1})$

\section{Component Aerodynamic Results for Superposition Method \#2}

The lift curves and drag polars for the fuselage, wings, braces, and tail empennage as well as the full configuration over a range of Mach number from 0.2 to 0.8 for the second superposition method \#2 are shown in Figs. 19 to 23.
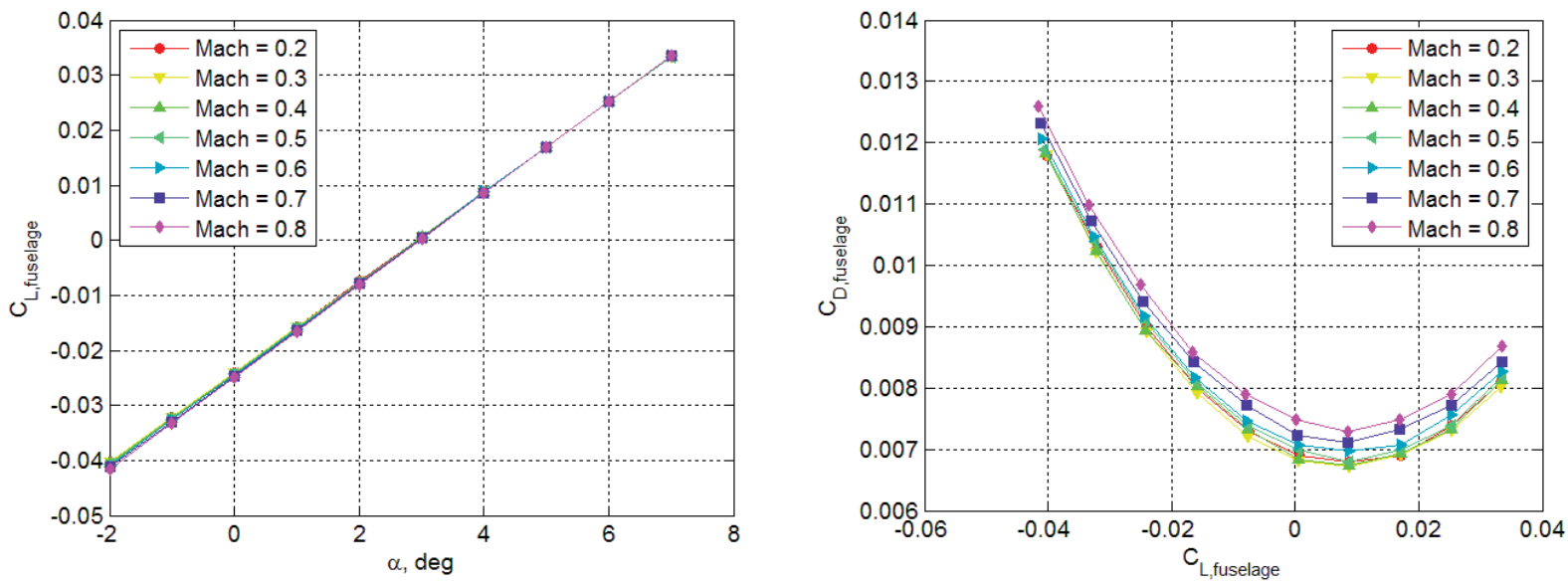

Figure 19. VORLAX Lift Curves and Drag Polars for TBW Fuselage 

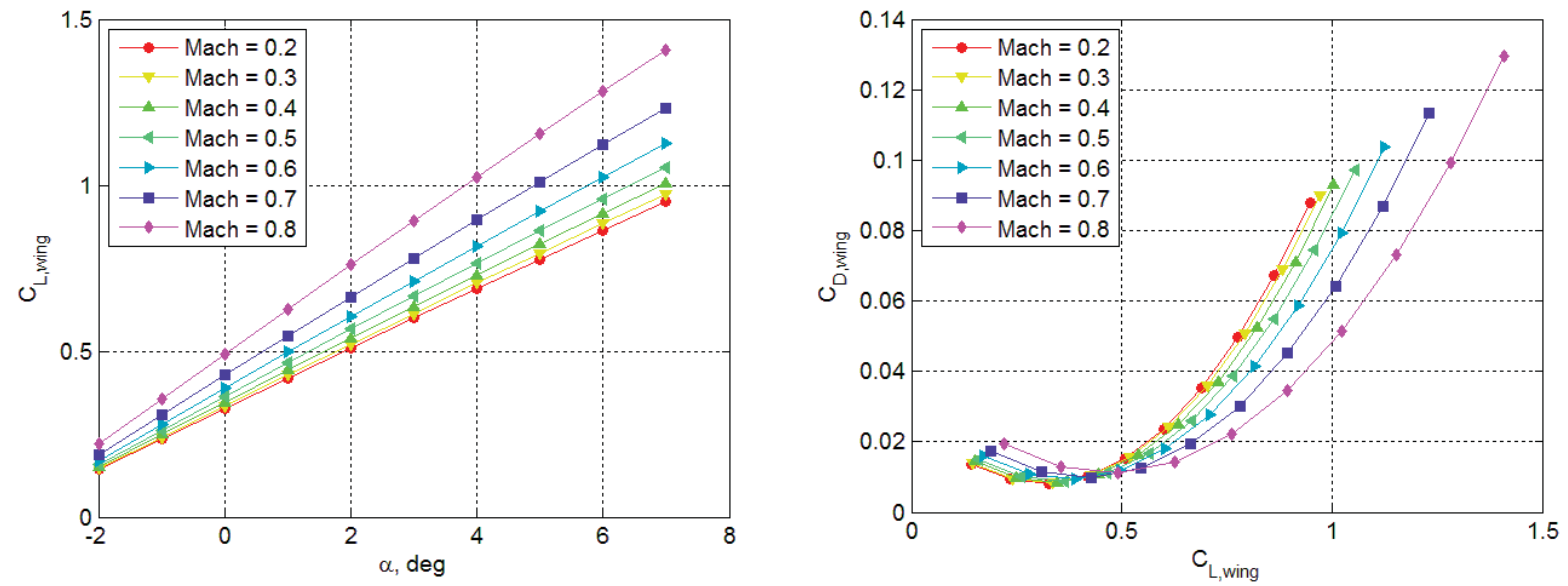

Figure 20. VORLAX Lift Curves and Drag Polars for TBW Wing
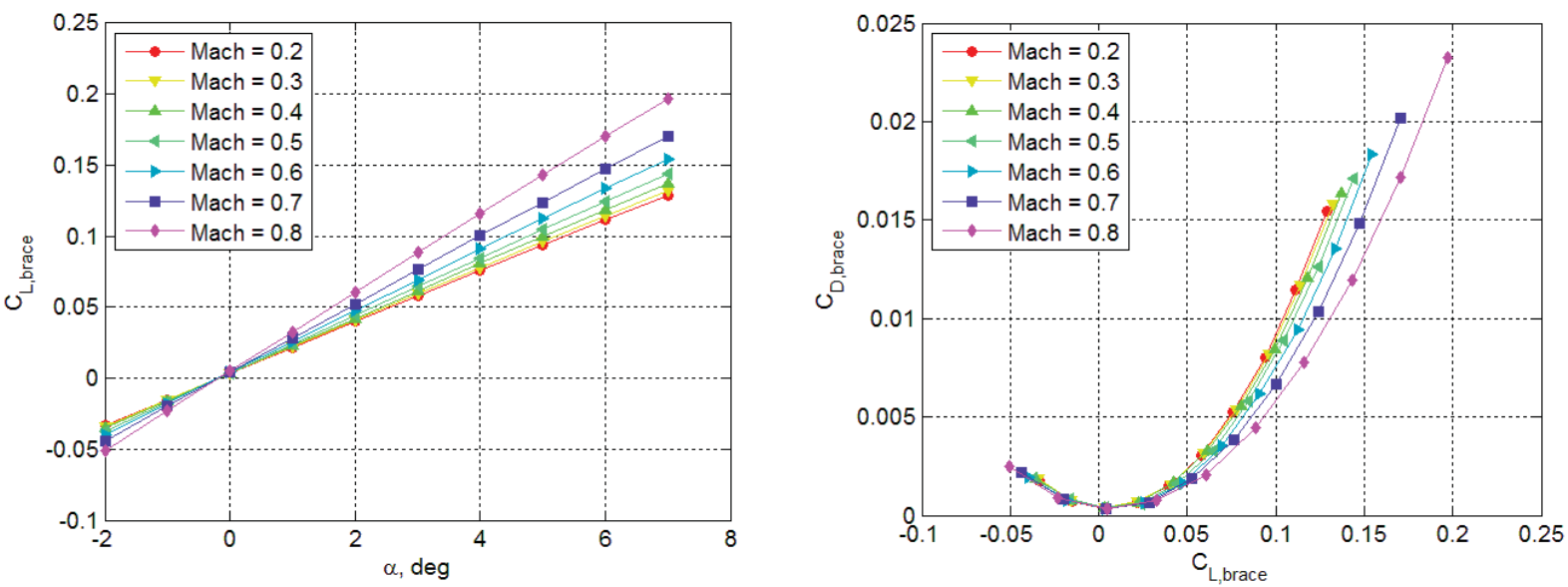

Figure 21. VORLAX Lift Curves and Drag Polars for TBW Brace
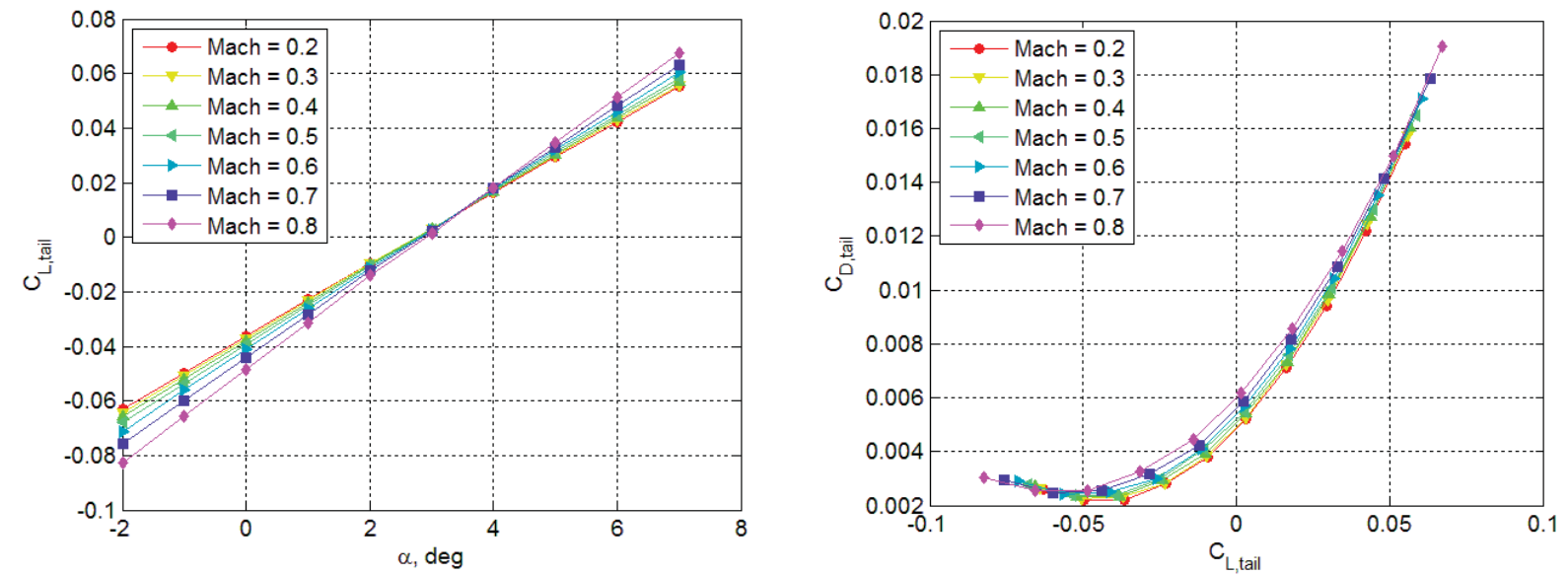

Figure 22. VORLAX Lift Curves and Drag Polars for TBW Tail Empennage 

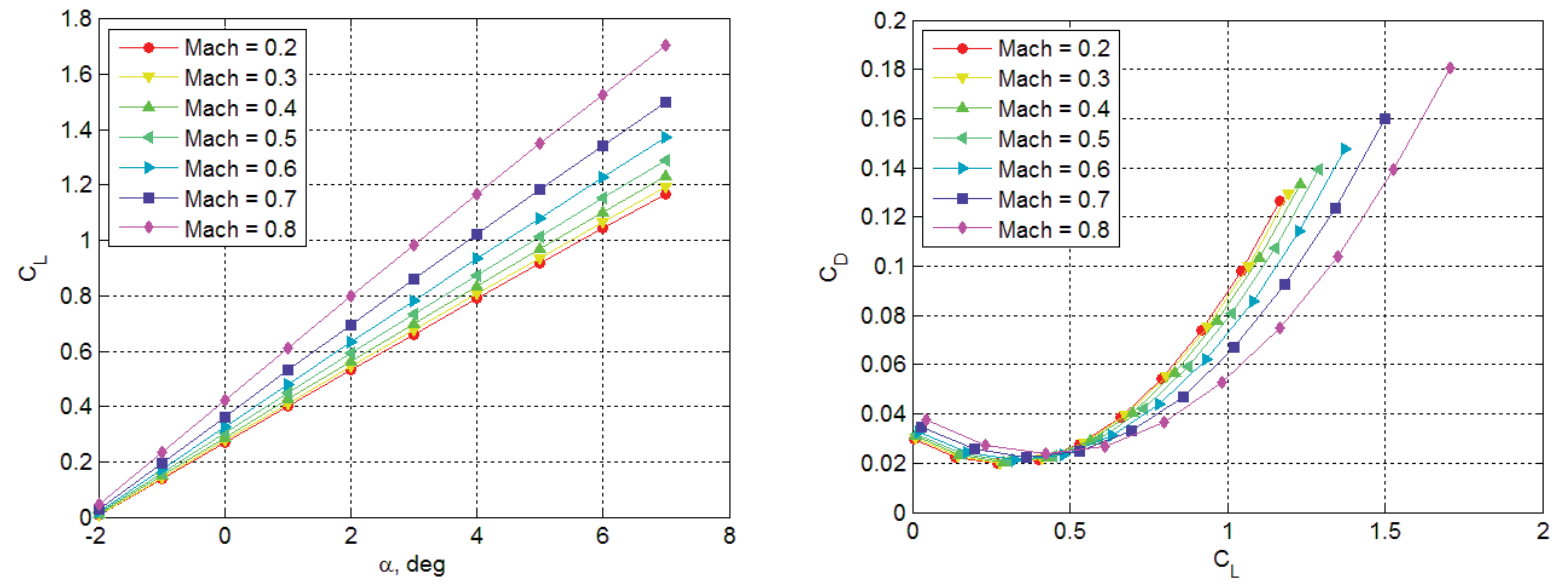

Figure 23. VORLAX Lift Curves and Drag Polars for TBW Full Configuration, Superposition Method \#2

At the design cruise condition of Mach 0.7 and altitude of 42,000 ft with $C_{L}=0.810$, the corresponding angle of attack is $\alpha=2.699^{\circ}$. At this angle of attack, the lift and drag contributions of the individual components to the total aerodynamics of the TBW are computed and shown in Tables 6, 7, and 8.

\begin{tabular}{|c|c|c|}
\hline & Component $C_{L}$ & \% Contribution \\
\hline \hline Wings & 0.7451 & 91.97 \\
\hline Braces & 0.0692 & 8.54 \\
\hline Tail Empennage & -0.0020 & -0.25 \\
\hline Fuselage & -0.0021 & -0.26 \\
\hline Total & 0.810 & 100 \\
\hline
\end{tabular}

Table 6. VORLAX Lift Contribution at Cruise $C_{L}=0.810$, Superposition Method \#2 $(1$ count $=0.0001)$

\begin{tabular}{|c|c|c|}
\hline & Component Induced $C_{D}$ (count) & \% Contribution \\
\hline \hline Wings & 240 & 72.07 \\
\hline Braces & 30 & 9.01 \\
\hline Tail Empennage & 44 & 13.21 \\
\hline Fuselage & 19 & 5.71 \\
\hline Total & 333 & 100 \\
\hline
\end{tabular}

Table 7. VORLAX Induced Drag Contribution at Cruise $C_{L}=0.810$, Superposition Method \#2 $(1$ count $=0.0001)$

\begin{tabular}{|c|c|c|}
\hline & Component Total $C_{D}$ (count) & \%Contribution \\
\hline \hline Wings & 270 & 62.65 \\
\hline Braces & 33 & 7.66 \\
\hline Tail Empennage & 54 & 12.53 \\
\hline Fuselage & 74 & 17.17 \\
\hline Total & 431 & 100 \\
\hline
\end{tabular}

Table 8. Total Estimated Drag Contribution at Cruise $C_{L}=0.810$, Superposition Method \#2 (1 count = 0.0001)

The wings generate about $92 \%$ of the total lift, while the trusses generate about $9 \%$ of lift. The tail empennage and fuselage generate down lift. Based on the model, the wings contribute almost $60 \%$ while the fuselage contributes about 
$17 \%$ to the total drag. The braces only contribute about $8 \%$ to the total drag, because its skin friction drag contribution calculated earlier in Table 1 is minimal due to the high transition Reynolds number used in this analysis.

\section{Discussion}

As an aerodynamic modeling tool, the computational efficiency of low-order aerodynamic methods such as vortexlattice and panel methods are suitable for conceptual aerodynamic analysis where medium fidelity is acceptable. In comparison to higher-fidelity CFD codes, VLM is a tool that is less computationally intensive and can be utilized readily in multidisciplinary analyses such as aeroelastic modeling. The development of the vortex-lattice aerodynamic capability pursued in this study is with the ultimate goal of developing a rapid aerodynamic modeling approach that can be coupled with structural finite-element models to model aeroelastic effects for future-generation aircraft concepts from the static aeroelasticity and flutter perspectives. The results of this study show that while the vortex-lattice approach is relatively fast, additional considerations must be taken in order to improve the modeling capability for complex aerodynamic configurations. While the aerodynamic superposition approach does show how to improve the aerodynamic prediction of VLM, the neglect of aerodynamic interference effects is a drawback and thus requires corrections from high-fidelity CFD tools.

In light of the difficulty posed by the complex configuration of the TBW, a panel method based on the panel code PANAIR is being considered for further expansion of the MDAO framework for the TBW. Considered as a conceptual aerodynamic method that is of higher order than VLM but simpler and faster than CFD, panel codes offer an alternative aerodynamic modeling approach that can still provide more rapid aerodynamic solutions in comparison to CFD. The PANAIR code, initially developed by Boeing for NASA, is a high-order panel code that solves potential flow problems for three-dimensional bodies. ${ }^{16}$ Panel codes discretize a full three-dimensional (3D) geometry into a series of panels, a clear advantage over vortex-lattice codes which reduce the 3D geometry of lifting surfaces down to mean camber surfaces. The deficiency of VLM in modeling blunt and non-lifting surfaces, such as a fuselage or nacelle, can be addressed by panel codes such as PANAIR. The use of PANAIR will complement the aerodynamic modeling capability of the MDAO framework to provide rapid aerodynamic and aeroelastic modeling of the TBW aircraft. Like VORLAX, however, the PANAIR code is still an inviscid code. Therefore, viscous drag and wave drag will need to be accounted for using other complementary techniques such as the integral boundary layer method. Both coupling of VORLAX and PANAIR with finite-element method to enable static aeroelasticity and dynamic aeroelasticity analyses are being developed for the MDAO framework.

\section{Conclusions}

This paper presents a recent aerodynamic analysis of the TBW aircraft using a conceptual vortex-lattice aerodynamic tool VORLAX. The deficiency of VLM in modeling the complex 3D geometry of the TBW using the full configuration geometry is observed in this study. Thus, an alternative approach based on the aerodynamic superposition method is investigated. The full configuration of the TBW is modeled by the superposition of aerodynamic solutions of individual components to estimate the total aerodynamic characteristics. This is an approximate method utilized for conceptual preliminary analysis, and aerodynamic interference effects occurring at the interfaces of components are not modeled in the superposition method. Excellent agreement in the lift curves between the VORLAX aerodynamic superposition method and the CFD results are demonstrated. The largest difference in the lift curve slope between the VORLAX aerodynamic superposition and the CFD methods is about $6 \%$, whereas the full configuration VORLAX model results in an error of almost $40 \%$. The drag polar predicted by the VORLAX aerodynamic superposition method is compared against the USM3D result, and agreement occurs between the two drag polars at a $C_{L}$ of about 0.8 . The aerodynamic contributions of individual components can be readily computed by the aerodynamic superposition method.

\section{Acknowledgments}

The authors would like to thank the Fixed Wing Project under the Fundamental Aeronautics Program of NASA Aeronautics Research Mission Directorate (ARMD) for funding support of this work. The authors also would like to acknowledge Robert Bartels and Richard Campbell at NASA Langley Research Center and John Melton at NASA Ames Research Center for providing CFD results of the Truss-Braced Wing configuration. 


\section{References}

${ }^{1}$ Bhatia, M., et. al.. "Structural and Aeroelastic Characteristics of Truss-Braced Wing: A Parametric Study," Journal of Aircraft, Vol 49, No. 1,2012

${ }^{2}$ Gundlach, J. F., Tetrault, P. A., Gern, F. H., Nagshineh-Pour, A. H., Ko, A., Schetz, J. A., et. al., "Conceptual Design Studies of a Strut-Braced Wing Transonic Transport," Journal of Aircraft, Vol. 37, No. 6, 2000

${ }^{3}$ Gur, O., Bhatia, M., Schetz, J.A., Mason, W. H., Kapania, R. K., and Mavris, D. N., "Design Optimization of a Truss-Braced Wing Transonic Transport Aircraft," Journal of Aircraft, Vol. 47, No. 6, 2010.

${ }^{4}$ Bradley, M. K. and Droney, C. K., "Subsonic Ultra Green Aircraft Research: Phase I Final Report," NASA Contractor Report NASA/CR2011-216847, Boeing Research and Technology, April 2011

${ }^{5}$ Bradley, M. K., Droney, C. K., and Allen, T. J., "Subsonic Ultra Green Aircraft Research Phase II: N+4 Advanced Concept Development," NASA Contractor Report NASA/CR-2012-217556, Boeing Research and Technology, May 2012

${ }^{6}$ Nguyen, N., "NASA Innovation Fund 2010 Project: Elastically Shaped Future Air Vehicle Concept," NASA Internal Report Submitted to NASA Innovative Partnerships Program Office, October 8, 2010.

${ }^{7}$ Urnes, Sr., J., Nguyen, N., Ippolito, C., Totah, J., Trinh, K., Ting, E., "A Mission-Adaptive Variable Camber Flap Control System to Optimize High Lift and Cruise Lift-to-Drag Ratios of Future N+3 Transport Aircraft," 51st AIAA Aerospace Sciences Meeting, AIAA-2013-0214, January 2013.

${ }^{8}$ Miranda, L. R., Elliott, R. D., Baker, W. M., “A Generalized Vortex Lattice Method for Subsonic and Supersonic Flow Applications,” NASA Contractor Report 2865, 1977

${ }^{9}$ Hahn, Andrew. S., "Vehicle Sketch Pad: A Parametric Geometry Modeler for Conceptual Aircraft Design," AIAA Aerospace Sciences Meeting, AIAA-2010-657, January 2010.

${ }^{10}$ Jordan, T. L., Langford, W. M., Belcastro, C. M., Foster, J. M., Shah, G. H., Howland, G., and Kidd, R., "Development of a Dynamically Scaled Generic Transport Model Testbest for Flight Research Experiments," AUVSI Unmanned Unlimited, Arlington, VA, 2004.

${ }^{11}$ Martin, G. L., "Paneling Techniques for Use with the Vorlax Computer Program," NASA Contractor Report NASA CR-145364, 1978

${ }^{12}$ Abbott, I. H. and Von Doenhoff, A. E., Theory of Wing Sections, Dover Publications, Inc., New York, pp. 97-103.

${ }^{13}$ Stanford University, AA241, http://adg.stanford.edu/aa241/drag/lsformfactor.html

${ }^{14}$ Stanford University, AA241, http://adg.stanford.edu/aa241/drag/BODYFORMFACTOR.HTML

${ }^{15}$ Hill, G., "Aerodynamic and Acoustic Investigations of an Advanced Over-the-Wing Nacelle Transport Aircraft Configuration," M.S. Thesis, Old Dominion University, May, 2007 (p. 35, Fig. 6).

${ }^{16}$ Derbyshire,T., Sidwell, K. W., "PAN AIR Summary Document (Version 1.0)," NASA Contrator Report 3250, Boeing Military Airplane Company, April 1982. 